OPEN ACCESS

Edited by: Gustavo Henrique Goldman, University of São Paulo, Brazil

Reviewed by: Jing Si,

Beijing Forestry University, China

Sanjay K. Singh,

Agharkar Research Institute, India

*Correspondence:

Alfredo Vizzin

alfredo.vizzini@unito.it

Specialty section:

This article was submitted to

Fungi and Their Interactions,

a section of the journal

Frontiers in Microbiology

Received: 06 February 2020

Accepted: 13 July 2020

Published: 11 August 2020

Citation:

Angelini $C$, Vizzini A, Justo $A$, Bizzi A, Davoli P and Kaya E (2020) First Report of a Neotropical Agaric (Lepiota spiculata, Agaricales, Basidiomycota) Containing Lethal $\alpha$-Amanitin at Toxicologically Relevant Levels. Front. Microbiol. 11:1833.

doi: 10.3389/fmicb.2020.01833

\section{First Report of a Neotropical Agaric (Lepiota spiculata, Agaricales, Basidiomycota) Containing Lethal $\alpha$-Amanitin at Toxicologically Relevant Levels}

\author{
Claudio Angelini ${ }^{1}$, Alfredo Vizzini ${ }^{2 *}$, Alfredo Justo ${ }^{3}$, Alberto Bizzi ${ }^{4}$, Paolo Davoli ${ }^{5}$ and \\ Ertuğrul Kaya ${ }^{6}$
}

${ }^{1}$ National Botanical Garden of Santo Domingo (JBSD), Santo Domingo, Dominican Republic, ${ }^{2}$ Dipartimento di Scienze della Vita e Biologia dei Sistemi, Università di Torino, Turin, Italy, ${ }^{3}$ New Brunswick Museum, Saint John, NB, Canada, ${ }^{4}$ Independent Researcher, Montecchio Maggiore, Italy, ${ }^{5}$ Independent Researcher, Vignola, Italy, ${ }^{6}$ Department of Pharmacology, Faculty of Medicine, Duzce University, Duzce, Turkey

A recent collection of Lepiota spiculata from the Dominican Republic is presented here. Macro- and micromorphological features of $L$. spiculata are described in detail, and its evolutionary (phylogenetic) position within Lepiota sect. Ovisporae, in the subincarnata/brunneoincarnata clade, is assessed on the basis of a combined $\mathrm{nrLSU}+\mathrm{nrlTS}+$ rpb2 + tef1 analysis. Additionally, high levels of deadly amatoxins were detected and quantified in L. spiculata for the first time by HPLC analysis; in particular, $\alpha$-amanitin was found at concentrations up to approximately $4 \mathrm{mg} / \mathrm{g}$ dry weight, which render $L$. spiculata a potentially lethal mushroom, if ingested.

Keywords: Agaricomycetes, Agaricaceae, Lepiota, molecular and chemical analyses, taxonomy, toxins, poisoning risk

\section{INTRODUCTION}

Lepiota spiculata was originally described by Pegler (1983) from the Lesser Antilles (Martinique Island), and since then it has been reported so far only from Mexico (Singer and Garcia, 1989). This would suggest that it is an uncommon or even rare mushroom. Despite scarce official reports, however, postage stamps from neotropical countries depicting L. spiculata (Saint Lucia, Saint Vincent and Grenadines) (Figure 1B) and photo vouchers from accredited websites (Baroni, 1998) from Puerto Rico (Figure 1B) and on social networks (from Trinidad and Tobago - J. Wong Sang, personal communication) (Figure 1B) indicate that this species might be more frequent in the Neotropics than we think. Vellinga (2004a) suggests that it is a neotropical species. The report of L. spiculata in China (Mao, 2000; Sysouphanthong et al., 2011) is dubious as the illustration and macroscopic and microscopic data presented in the book "The Macrofungi in China" (Mao, 2000) suggest that this collection likely represents a separate, unknown lepiotoid species (in agreement with Vellinga EC - personal communication). All reports of L. spiculata are on abandoned termite nests (Pegler, 1983; Singer and Garcia, 1989; Vellinga, 2004a), usually attached to the base of deciduous trees. Our own recent collection from the Dominican Republic described here was growing on an abandoned and decayed nest of the termite Nasutitermes corniger Motschulsky (= N. costalis Holmgren), locally referred as "comején" (Figure 1A). 

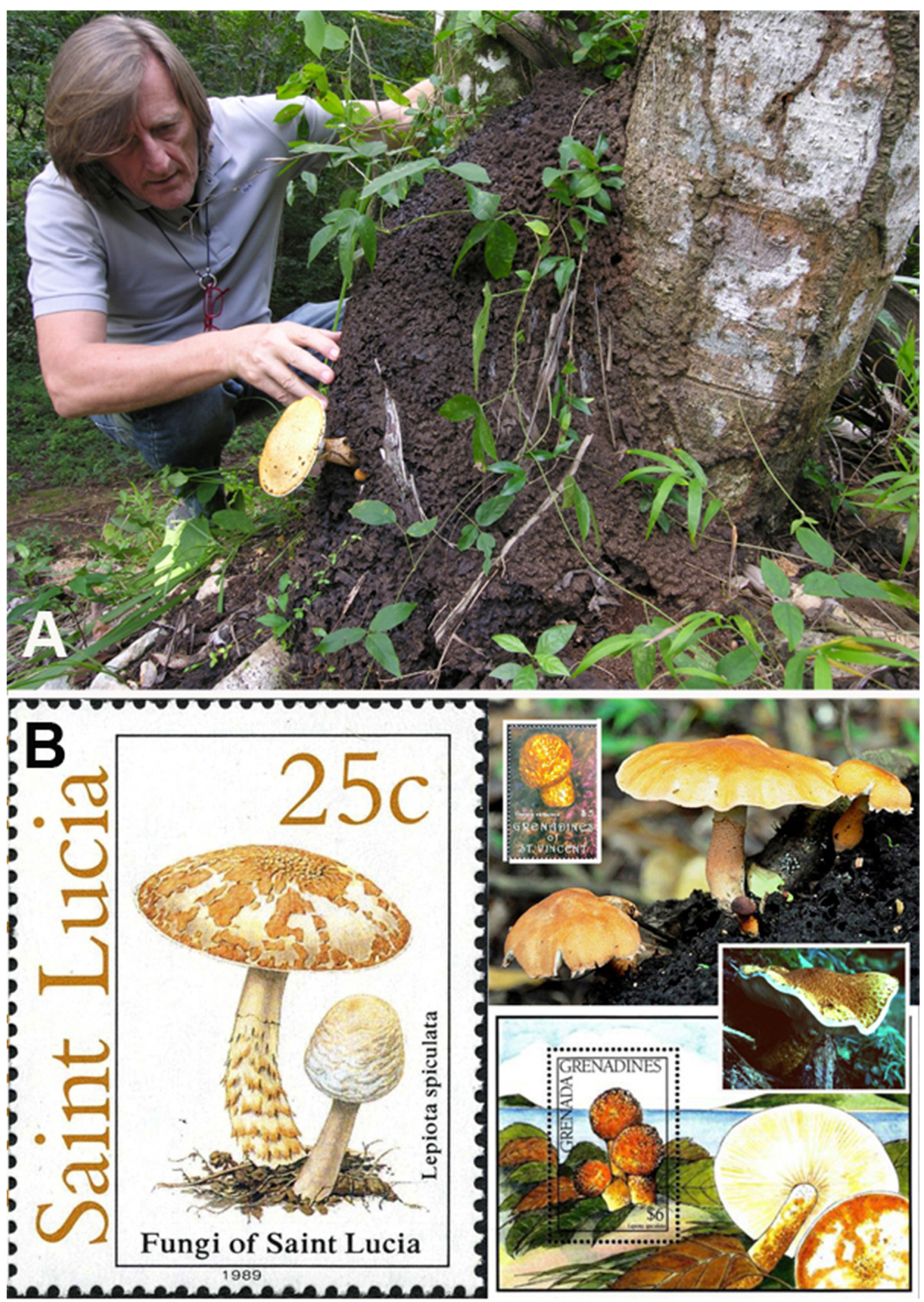

FIGURE 1 | Lepiota spiculata. (A) Basidiomes in habitat growing on a termite nest (photo by C. Angelini). (B) Representations on stamps and websites. Person in image is the first author. 
This collection represents the first official report of L. spiculata from the Greater Antilles (Hispaniola Island, Dominican Republic) and allowed us to document its morphology in different growth stages (from primordium to mature basidiome) and to clarify its evolutionary (phylogenetic) position by analyzing two molecular datasets: a combined nrLSU (28S) + nrITS + rpb2 + tef1 dataset and a nrITSonly dataset. Lepiota spiculata was found in our phylogenetic analyses to be part of Lepiota sect. Ovisporae (J.E. Lange) Kühner, in the subincarnata/brunneoincarnata clade, which is known to harbor amatoxin-containing species (Vellinga, 2001, 2003; Zhang et al., 2019). Based on this taxonomic placement, we hypothesized that L. spiculata might also produce amatoxins and could therefore be considered a poisonous species. A positive outcome of the Wieland-Meixner spot test (Meixner, 1979) on our specimens hinted at the occurrence of hydroxy-substituted indole-containing metabolites in the basidiomes. Subsequent chemical analysis by means of HPLCMS analysis confirmed unequivocally the presence of amatoxins; in particular, $\alpha$-amanitin was found to reach levels of $3.9 \mathrm{mg} / \mathrm{g}$ dry weight in the pileus, which render L. spiculata a potentially lethal mushroom species, if consumed.

\section{MATERIALS AND METHODS}

\section{Fungal Material Collection}

Specimens examined (one adult and one at the primordium stage) were collected in a hilly forest near the Sosúa cemetery in the Puerto Plata province, Dominican Republic, and are deposited at the Dr. Rafael Ma. Moscoso National Botanical Garden herbarium (JBSD). Institutional herbarium acronyms follow Index Herbariorum (Thiers, 2020), while "ANGE” refers to the personal herbarium of CA.

\section{Morphological Studies}

Macroscopic and microscopic descriptions are based exclusively on the recent collection made in the Dominican Republic. Color terms in capital letters (e.g., ORANGE 226, pl. XVI) are from Séguy (1936). Terminology for descriptive terms is according to Vellinga $(1988,2001)$. Photographs of collections were taken in the natural habitat using a Nikon Coolpix 8400 digital camera. Microscopic anatomical features were observed and recorded from revived dried material; sections were rehydrated either in water or in $5 \%$ potassium hydroxide $(\mathrm{KOH})$. All microscopic structures were observed and measured from preparations in anionic Congo Red. Colors and pigments were described after examination in water. Measurements were made at $1000 \times$ using a calibrated ocular micrometer (Meiji MT4310H optical light microscope). Basidiospores were measured directly from the hymenophore of mature basidiomes. The notation $[\mathrm{n} / \mathrm{m} / \mathrm{p}]$ indicates that measurements were made on " $n$ " randomly selected basidiospores from " $m$ " basidiomes of " $p$ " collections - $\mathrm{Q}=$ length/width ratio, $\mathrm{Qm}=$ average quotient (length/width ratio) - and the average spore volume $(\mathrm{V})$ was approximated as a rotation ellipsoid $[\mathrm{V}=(\pi . \mathrm{L} . \mathrm{W} 2) / 6]$. The width of each basidium was measured at the widest part, and the length was measured from the apex (sterigmata excluded) to the basal septum. Metachromatic and iodine reactions were tested by staining the basidiospores in Brilliant cresyl blue and Melzer's reagent, respectively. Line drawings of microstructures were traced in freehand based on digital photomicrographs of rehydrated material.

\section{DNA Extraction, PCR Amplification, and DNA Sequencing}

Total DNA was extracted from dry specimens employing a modified protocol based on Murray and Thompson (1980). PCR amplification was performed with the primers ITS1F and ITS4 (White et al., 1990; Gardes and Bruns, 1993) for the nrITS region, while LR0R and LR5 (Vilgalys and Hester, 1990; Cubeta et al., 1991) were used to amplify the 28S rDNA region, EF1-983F and EF1-1567R (Rehner and Buckley, 2005) for the translation elongation factor 1a (TEF1) gene, and finally bRPB2-6F and bRPB2-7R2 for the RNA polymerase II second largest subunit (RPB2) gene (Liu et al., 1999; Matheny et al., 2007). PCR reactions were performed under a program consisting of a hot start at $95^{\circ} \mathrm{C}$ for $5 \mathrm{~min}$, followed by 35 cycles at $94^{\circ}, 54^{\circ}$, and $72^{\circ} \mathrm{C}\left(45,30\right.$, and $45 \mathrm{~s}$, respectively) and a final $72^{\circ} \mathrm{C}$ step for $10 \mathrm{~min}$. PCR products were checked in 1\% agarose gels, and positive reactions were sequenced with one or both PCR primers. Chromatograms were checked searching for putative reading errors, and these were corrected.

The sequences were submitted to GenBank ${ }^{1}$, and their accession numbers are reported in Table $\mathbf{1}$.

\section{Sequence Alignment, Data Set Assembly, and Phylogenetic Analyses}

A four-gene dataset (nrLSU, nrITS, $r p b 2$, and tef1) was assembled, which includes a wide sampling of the agaricoid genera in the Agaricaceae (Sysouphanthong et al., 2011). All species of Lepiota (Pers.) Gray with available rpb2 data in GenBank were included in the analysis. The final dataset includes 18 species in the Agaricaceae, with Amanita brunnescens G.F. Atk. and Pluteus aff. romellii chosen as outgroup taxa. Sequences used in the analyses are listed in Table 1. Separately, we assembled an nrITS dataset that includes a wide representation of the different lineages of Lepiota sensu Vellinga (2003, 2004b), including also species of Lepiota sect. Echinatae Fayod, Cystolepiota Singer, and Melanophyllum Velen. The final dataset consists of 243 nrITS sequences, and the midpoint rooting option was chosen to root the tree. All sequences were aligned using MAFFT version 7 (Katoh and Toh, 2008), and the strategy FFT-NS-i was selected. Alignments were inspected and manually corrected using AliView (Larsson, 2014). Maximum likelihood (ML) analyses were run using RAxML version 8.2.10 (Stamatakis, 2014), under a GTR model with one hundred rapid bootstrap (BS) replicates. The ML analyses were run using the resources at the CIPRES Science Gateway (Miller et al., 2010).

\footnotetext{
${ }^{1}$ http://www.ncbi.nlm.nih.gov/genbank/
} 
TABLE 1 | Sequences used in the combined phylogenetic analyses. Newly generated sequences are boldfaced.

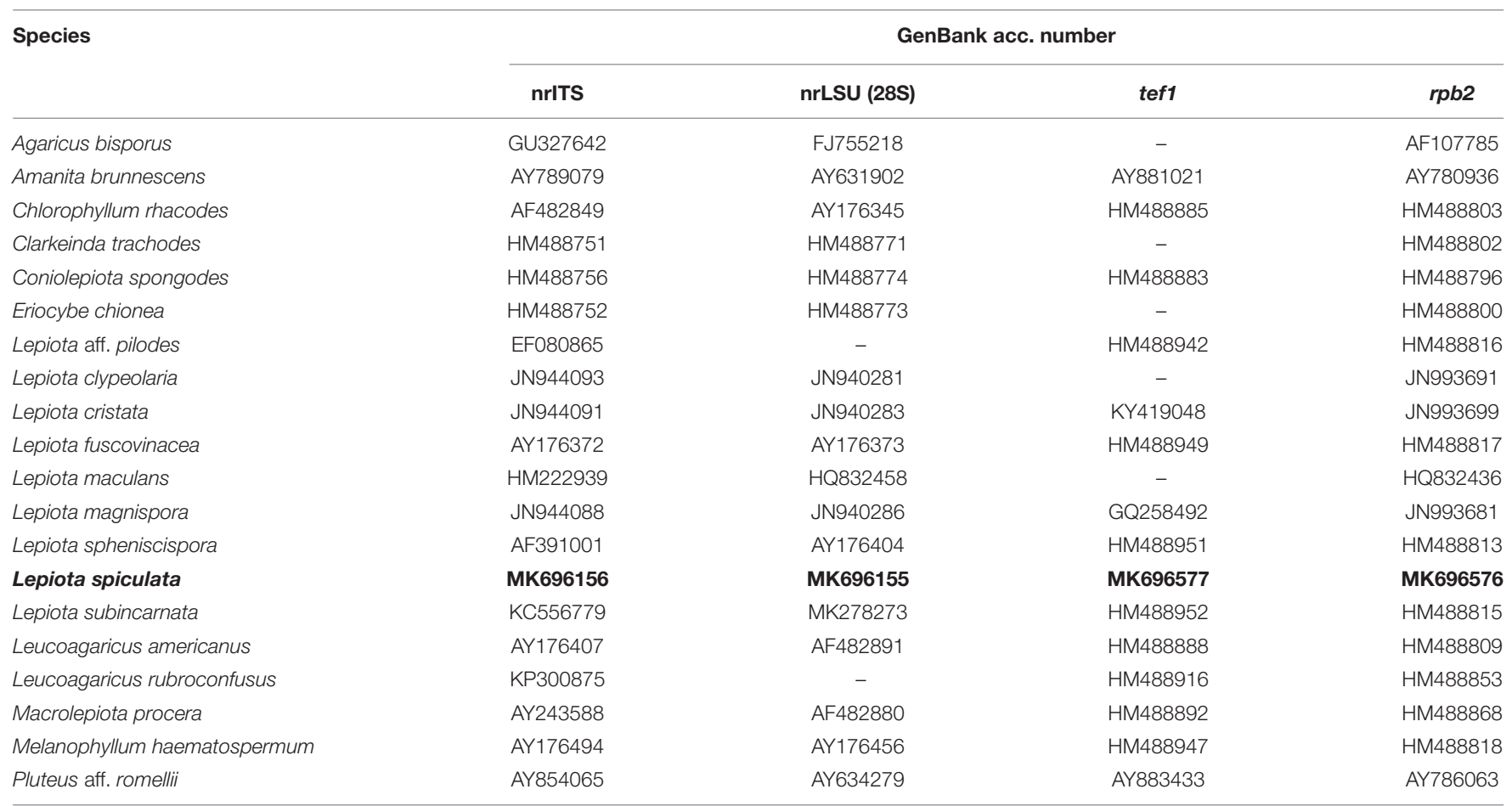

\section{Wieland-Meixner Test}

A small fragment of dried L. spiculata was rehydrated with water and squeezed to obtain a drop of mushroom juice that was spotted onto a piece of lignin-containing paper and left to evaporate at room temperature. Two drops of concentrated hydrochloric acid $(37 \% \mathrm{w} / \mathrm{w})$ were placed directly onto the resulting spot and left to react at room temperature away from direct sunlight. The appearance of a bluish-green color within a few minutes indicated a positive result for the Wieland-Meixner spot test (Meixner, 1979; Beutler and Vergeer, 1980; Walton, 2018). As the negative control, a drop of concentrated $\mathrm{HCl}$ was spotted alone onto the paper matrix.

\section{Chemical Analysis}

Prior to chemical analysis, all samples of $L$. spiculata were further dried at $55^{\circ} \mathrm{C}$ under airflow for $24 \mathrm{~h}$. Pileus and stipe were analyzed separately. Dried mushroom samples were ground and homogenized in methanol/water/0.01 $\mathrm{M} \mathrm{HCl}(5: 4: 1 \mathrm{v} / \mathrm{v} / \mathrm{v})$ as the extraction solvent using a tissue homogenizer and a 1:30 $(\mathrm{w} / \mathrm{v})$ sample-to-solvent ratio. After $24 \mathrm{~h}$ extraction, samples were centrifuged for $10 \mathrm{~min}$ at $5000 \mathrm{rpm}$ and the supernatant was filtered through a $0.22 \mu \mathrm{m}$ syringe filter, and $10 \mu \mathrm{L}$ aliquots of the resulting mushroom extracts were subjected to reversedphase HPLC analysis. Standards for $\alpha$-amanitin and phalloidin were obtained from Sigma-Aldrich (United States), whereas $\beta$-amanitin, $\gamma$-amanitin, and phallacidin were from Enzo Life Sciences (Farmingdale, NY, United States). All solvents used were HPLC grade. Stock solutions of all standards at $100 \mu \mathrm{g} / \mathrm{mL}$ were prepared in methanol. Calibration standards were diluted in the extraction solvent at concentrations of 1, 10, 20, 100, 200, and $1000 \mathrm{ng} / \mathrm{mL}$; for each standard calibration, curves were linear over the range of interest $\left(r^{2}>0.99\right)$. Mushroom extracts were analyzed in triplicate; data are given as $\mathrm{mg}$ toxin per g dried mushroom (mean \pm standard deviation) for each sample.

Separation and quantification of toxins in samples of L. spiculata was performed by reversed-phase HPLC analysis following well established procedures (Yilmaz et al., 2013, 2015, 2018). A Shimadzu instrument (Japan) fitted with an RP-C18 column $(5 \mu \mathrm{m}$ particle size; $150 \mathrm{~mm} \times 4.6 \mathrm{~mm}$; Agilent Technologies, Palo Alto, CA, United States) was used and equipped with an online diode-array detector (DAD). Isocratic elution with $0.05 \mathrm{M}$ ammonium acetate $(\mathrm{pH} 5.5$ with acetic acid)/acetonitrile 90:10 (v/v) as the mobile phase was used at a flow rate of $1 \mathrm{~mL} / \mathrm{min}$ at $40^{\circ} \mathrm{C}$. Detection was performed at $303 \mathrm{~nm}$ for amatoxins and at $290 \mathrm{~nm}$ for phallotoxins, and UV absorption spectra were recorded online with the photodiodearray detection system. Detection limits were determined to be $2.5 \mathrm{ng} / \mathrm{g}$ dry weight for amatoxins and $2.8 \mathrm{ng} / \mathrm{g}$ dry weight for phallotoxins.

In order to confirm unambiguously the identity of the toxins, mass spectra of the relevant peaks in mushroom extracts were recorded with a Shimadzu (Japan) 8040 MS/MS instrument equipped with an ESI source operating at a spray voltage of $3 \mathrm{kV}$ in the ESI-positive ionization mode, following LC-MS/MS conditions similar to those reported by Gicquel et al. (2014). The capillary temperature was $350^{\circ} \mathrm{C}$, and nitrogen was supplied as drying gas with a pressure of 35 p.s.i.g $(1$ p.s.i. $=6894.76 \mathrm{~Pa})$. Data were acquired in targeted single ion monitoring (SIM) mode and scanning mode simultaneously. Tuning parameters 
were optimized by direct infusion of individual standards at a concentration of $1 \mu \mathrm{g} / \mathrm{mL}$ in the mobile phase into the ionization probe at a flow rate of $0.4 \mathrm{~mL} / \mathrm{min}$ in the ESI-positive mode.

\section{RESULTS}

\section{Taxonomy}

Lepiota spiculata Pegler, Kew Bulletin, Additional

Series 9: 390, 1983.

(Figures 1-3)

\section{Description}

Pileus $7 \mathrm{~cm}$ in diam., spherical-globose, then hemispherical, finally expanding to plano-convex (Figures 2B-D); surface at first formed by a thick brown-ochre (ORANGE 176, Pl. XII) layer of wooly appearance (which covers the whole basidiome in the primordium), then consisting of pyramidal warts with yellow-orange curved apices (ORANGE 196, Pl. XIV) with reddish tips (ORANGE 151, Pl. XI) that completely cover the pileus, at complete maturity ochraceous buff (ORANGE 211, Pl. XV) with the brownish apices (ORANGE 176, Pl.VII) covering above all the central part, while toward the periphery the layer tends to rise up to the margin and then breaks into irregular squamules on a white background (Figure $\mathbf{2 H}$ ). Margin strongly inrolled in the primordium almost completely curling over the lamellae (Figure 2A). Lamellae free, or subfree-annexed, never distant, white (BLANC ABSOLU, Pl. XLIX), lilac-vinaceous when bruised (ROUGE 11, Pl. I) (Figure 2G), quite thick, ventricose, 6-7.5 mm wide, with the edge slightly eroded, lamellulae of various lengths and usually 1-2 between each pair of lamellae reaching the stipe. Stipe $8 \times(1-) 1.5(-$ 3) $\mathrm{cm}$, cylindrical, attenuated at the apex (1 cm wide), with the base swollen ( $3 \mathrm{~cm}$ wide) and rooting, solid, at the apex white and smooth, more scaly below, ochre-brownish (ORANGE 176, Pl.VII); all parts lilac-vinaceous on bruising (Figure 2E). Annulus white, evident only in the primordium, then fragile, fugacious, almost absent, separating the apical glabrous part and the lower scaly part of the stipe. Context very thick, up to $1 \mathrm{~cm}$ at the disk, white, unchanging on exposure, or lilacvinaceous when bitten by insects or mollusks (Figure $2 \mathrm{~F}$ ); odor none; taste mild, subfarinaceous. Basidiospores [50/1/1] (5.0-) 5.5-8.0 $\times 3.5-4.5 \mu \mathrm{m}$, on average $6.5 \pm 0.7 \times 4.1 \pm 0.2 \mu \mathrm{m}$, $\mathrm{Q}=1.38-2.00, \mathrm{Qm}=1.60, \mathrm{~V}=57 \mu \mathrm{m}^{3}$, ellipsoid to oblong, thin-walled, hyaline, dextrinoid, not metachromatic, with wall that does not swell in either ammonia or acetic acid. Basidia 18-36 × 6-7 $\mu \mathrm{m}$, cylindrical-clavate, tetrasporic. Lamella edge sterile, with crowded cheilocystidia. Cheilocystidia 20.5-34 × 10-13.5 $\mu \mathrm{m}$, clavate, subfusiform, hyaline, thinwalled. Pleurocystidia absent. Pileus covering a conspicuous but discontinuous trichoderm of erect hairs, consisting of hyphae 65-175 $\times 10-22.5 \mu \mathrm{m}$ wide, gathered in thin needle-like tufts, flexuous, often irregular, with tapered or clavate apices, with thickened walls with yellow-ochre (VERT337, Pl. XXIII) parietal pigment. Stipitipellis a cutis consisting of cylindrical, 4-10 $\mu \mathrm{m}$ wide hyphae, with squamules as on pileus. Clamp-connections abundant, especially at the base of the cheilocystidia and on the hyphae of the pileus covering and context (see microscopic drawings in Figure 3A).

\section{Material Studied}

Dominican Republic, municipality of Sosúa, Puerto Plata province, local cemetery, $19^{\circ} 44^{\prime} 4^{\prime \prime} \mathrm{N}, 70^{\circ} 32^{\prime} 21^{\prime \prime} \mathrm{E}, 50 \mathrm{~m}$ a.s.l., two specimens (one adult and one at the primordium stage) growing on an abandoned termite nest, 06 Dec. 2017 - Leg. C. Angelini, Det. E.C.Vellinga. (JBSD127426, ANGE971) (Figure 1A).

\section{Phylogenetic Position of the Species}

In the 4-gene dataset (Figure 4), L. spiculata appears as the sister species to L. subincarnata J.E. Lange with high support. Both species are part of a more inclusive clade with Lepiota aff. pilodes. The genus Lepiota, sensu Vellinga (2003), receives moderate support in this dataset, either with or without the inclusion of Melanophyllum Velen.

The nrITS sequence of Lepiota spiculata is located in the nrITS analysis as part of the Lepiota Clade $2 \mathrm{a}$ in the sense of Vellinga (2003), even though this placement does not receive high support in the analyses (Figure 5). This clade includes species traditionally placed in Lepiota subsect. Helveolinae Bon \& Boiffard, i.e., L. brunneoincarnata Chodat \& C. Martín, L. subincarnata J.E. Lange, L. elaiophylla Vellinga \& Huijser (Vellinga, 2001), all known to contain amatoxins (Vellinga, 2001; Sgambelluri et al., 2014; Walton, 2018). Two amatoxincontaining species recently described from China, Lepiota venenata Zhu L. Yang \& Z.H. Chen (Cai et al., 2018) and Lepiota subvenenata Hai J. Li, Y.Z. Zhang \& C.Y. Sun (Zhang et al., 2019), are also placed in Clade 2a (Figure 5). Lepiota brunneosquamula J.F. Liang \& Z.L. Yang appears as sister to L. subvenenata (Figure 5), and while the presence of amatoxins in this species has been suggested due to its phylogenetic placement (Zhang et al., 2019), it has yet to be confirmed by chemical analyses.

\section{Wieland-Meixner Test}

The Wieland-Meixner test on dried specimens of L. spiculata resulted in a clear positive reaction, viz., a blue-green color (Figure 3B), thereby leading support to our initial surmise. The Wieland-Meixner spot test is most useful and straightforward to detect the presence of hydroxylated indole derivatives such as, for instance, amatoxins (e.g., $\alpha-, \beta-, \quad \gamma-, \varepsilon$-amanitin and amanullin); likewise, however, it also detects 4- and 5-substituted tryptophan derivatives such as psilocin ( $N, N$-dimethyl-4-hydroxytryptamine), bufotenin (N,N-dimethyl-5-hydroxytryptamine), serotonin (5-hydroxytryptamine), and 5-hydroxytryptophan (Beutler and Vergeer, 1980; Walton, 2018). In the case of 5hydroxytryptamines, the positive Wieland-Meixner spot test is preceded by an initial discoloration to reddishbrown colors, whereas 4-hydroxytryptamines are reported to display a preliminary shift to gray hues before discoloring ultimately to blue-green (Beutler and Vergeer, 1980). Since lepiotaceous fungi are not known to produce 4or 5-hydroxylated tryptophan-derived metabolites, and given the fact that the Wieland-Meixner spot test on 

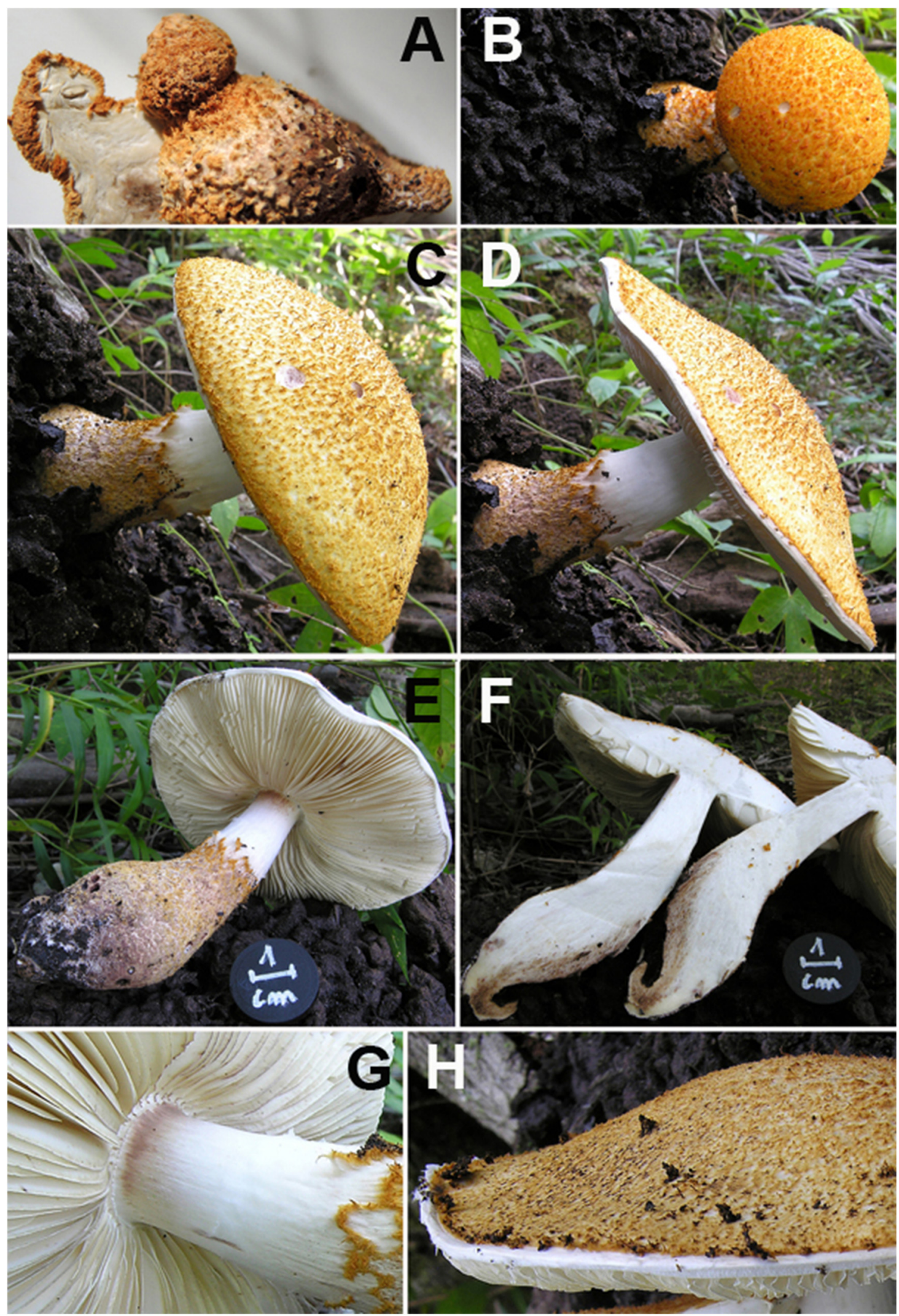

FIGURE 2 | Lepiota spiculata. Macroscopic characters. (A) Primordium in section. (B-D) Pileus in various stages of development. (E) Stipe. (F) Basidiome in section. (G) Lamellae. (H) Pileus surface at maturity (photos by C. Angelini). 


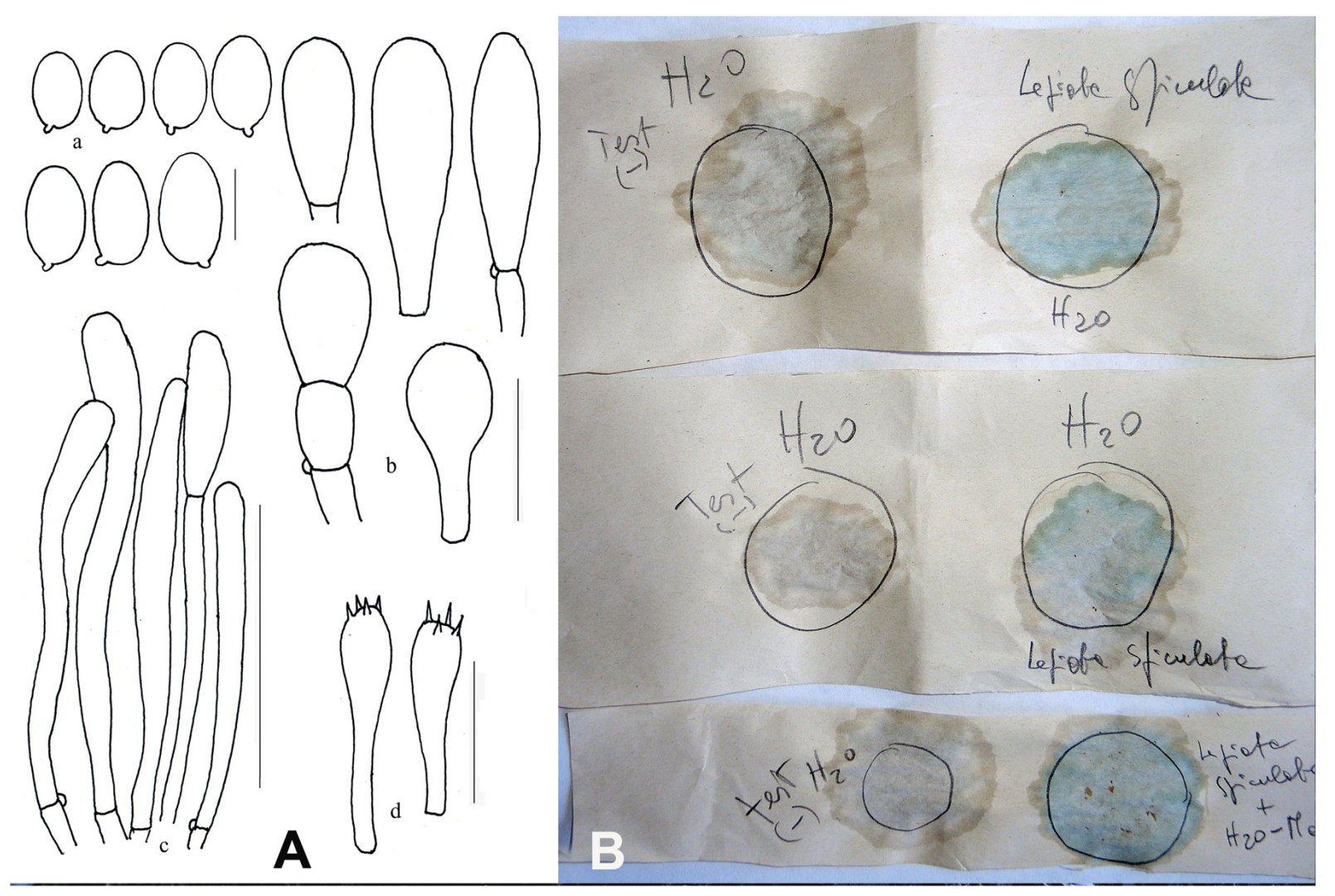

FIGURE 3 | Lepiota spiculata. (A) Ink drawing of microscopical traits. Bars: (a) spores $5 \mu \mathrm{m}$; (b) cheilocystidia $20 \mu \mathrm{m}$; (c) pileipellis $100 \mu \mathrm{m}$; (d) basidia $20 \mu \mathrm{m}$ (drawings by A. Bizzi). (B) Wieland-Meixner test: negative control (left column) and blue-green positive reaction (right column) (photo by C. Angelini).

L. spiculata did not show any preliminary discoloration to either reddish-brown or gray colors, this suggested that the direct reaction to blue-green was likely due to the presence of amatoxins.

\section{Chemical Analysis}

Analysis of mushroom extracts by means of reversed-phase HPLC-UV-DAD and LC-MS/MS confirmed unequivocally that L. spiculata contains $\alpha$-amanitin (Figure 6); the concentration of the toxin is highest in the pileus, where a level of $3.9 \mathrm{mg} / \mathrm{g}$ dry weight was measured (Table 2). $\alpha$-Amanitin gave the expected $[\mathrm{M}+\mathrm{H}]^{+}$ion at $m / z 919.35$ by LC-MS/MS ESI analysis in the positive ionization mode (Figure 6). $\beta$-Amanitin, $\gamma$-amanitin, phalloidin, and phallacidin were not detected in either pileus or stipe (Figure 6). Interestingly, an additional minor peak that did not correspond to any of the toxin standards used was detected in extracts of L. spiculata (Figure 7); this peak eluted earlier than $\alpha$-amanitin under the RP-HPLC conditions employed $\left(t_{\mathrm{R}} 14.9\right.$ vs. $15.7 \mathrm{~min}$, respectively). The UV spectrum for the unknown peak is reported in Figure $\mathbf{8}$ and resembles a phallotoxin-type spectrum, but in the absence of additional spectroscopic data its identity must remain unanswered at present. By analogy to $\alpha$-amanitin, the level of the unknown toxin is higher in the pileus of L. spiculata, where it reached $1.2 \mathrm{mg} / \mathrm{g}$ dry weight (expressed as $\alpha$-amanitin equivalent) (Table 2 ).

\section{DISCUSSION}

Lepiota spiculata was described by Pegler based on a collection by Fiard in Martinique. The original description includes two in situ photographs and one ink drawing from the holotype with macroscopical and microscopical traits (Pegler, 1983). The exsiccatum of the holotype was deposited in Kew (K) along with the drawing by Fiard himself (this drawing no longer exists, either at K, or in LIP herbaria - personal communications). Our collection from the Dominican Republic matches exactly with macroscopic and microscopic traits of the original collection from Martinique (Pegler, 1983). The only difference in the Dominican collection is the almost total absence of a true annulus (partial veil) on the stipe (Figure 2G), which is clearly visible only in the section of the primordium (Figure 2A).

Based on the few collections made (Pegler, 1983; Singer and Garcia, 1989; our report), L. spiculata appears to be a strictly termitophilic species. However, despite a well-established body of knowledge on the symbiotic relationships between paleotropical fungus-growing termites (Macrotermitineae) and species of the genus Termitomyces R. Heim (Agaricales, Lyophyllaceae; Aanen et al., 2007; Nobre et al., 2011; Ye et al., 2019) and other associated fungi (Visser et al., 2011), the relationship between neotropical L. spiculata and termites, if any, remains unknown and warrants further ecological investigations. 


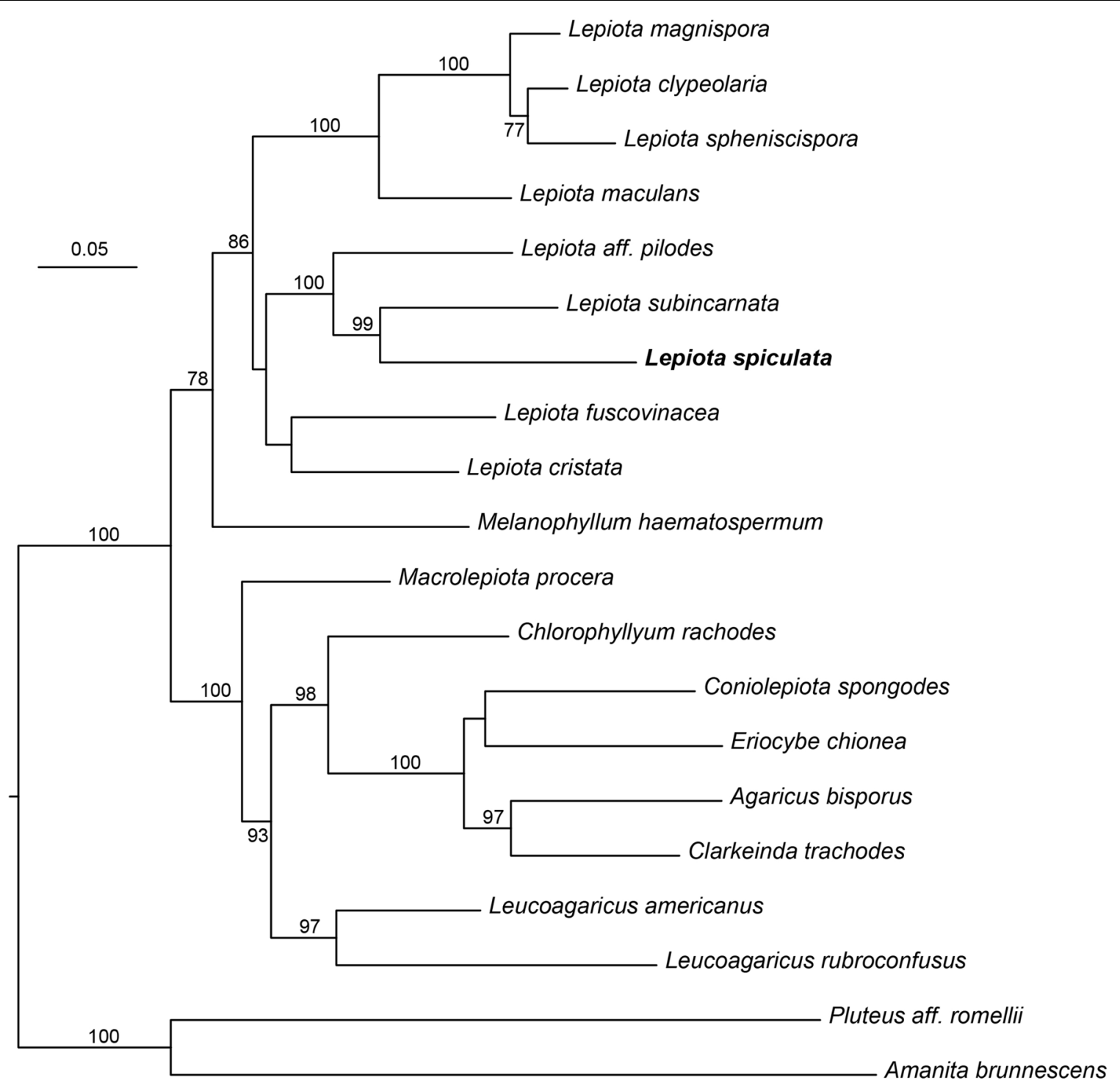

FIGURE 4 | Phylogenetic position of Lepiota spiculata based on a Maximum likelihood analysis of a combined nrlTS/nrLSU/tef1/rpb2 data set.

After considering L. spiculata as a species of dubious position in Lepiota at section level because of its mediumsized basidiomes, robustness, and well-developed cheilocystidia, Pegler (1983) placed it doubtfully in Lepiota sec. Ovisporae because of its ovo-ellipsoid spores and the trichodermic pileipellis with clavate-cylindrical terminal elements. He also recognized affinities with Lepiota species of the sect. Echinatae Fayod emend. Knudsen [= genus Echinoderma (Locq. ex Bon) Bon] due to the presence of pyramidal verrucae on pileus surface. Interestingly, the present molecular phylogenetic analyses (Figures 4, 5) confirm its placement in sect. Ovisporae, close to L. subincarnata J.E. Lange (= L. josserandii Bon \& Boiffard) and L. brunneoincarnata Chodat \& C. Martín. The massive basidiomes of L. spiculata are not typically lepiotoid, and it might well be confused with another genus (Floccularia Pouzar or Amanita Pers.). Because of its pleasant taste and lack of unpleasant odor, L. spiculata could be easily mistaken for an edible mushroom with severe health consequences if consumed due to the presence of $\alpha$-amanitin in toxic concentrations. In fact, $\alpha$-amanitin represents the major toxin in L. spiculata, where levels of approximately $4 \mathrm{mg} / \mathrm{g}$ dry weight are reached in the pileus, whereas $\beta$-amanitin, $\gamma$-amanitin, phalloidin, and phallacidin are absent; an additional phallotoxin-like toxin is also present in conspicuous amounts, especially in the pileus, but no identification has been attempted in the present work. In Lepiota, the pileus seems richer in $\alpha$-amatoxin than the stipe as suggested by our data (Table 2) and previous analyses of L. brunneoincarnata (Yilmaz et al., 2015; Sun et al., 2019). Such high levels of $\alpha$-amanitin in L. spiculata are comparable to those reported in the literature for poisonous Lepiota species belonging to the same section and clade, such as L. subincarnata and L. brunneoincarnata (Enjalbert et al., 2002; 


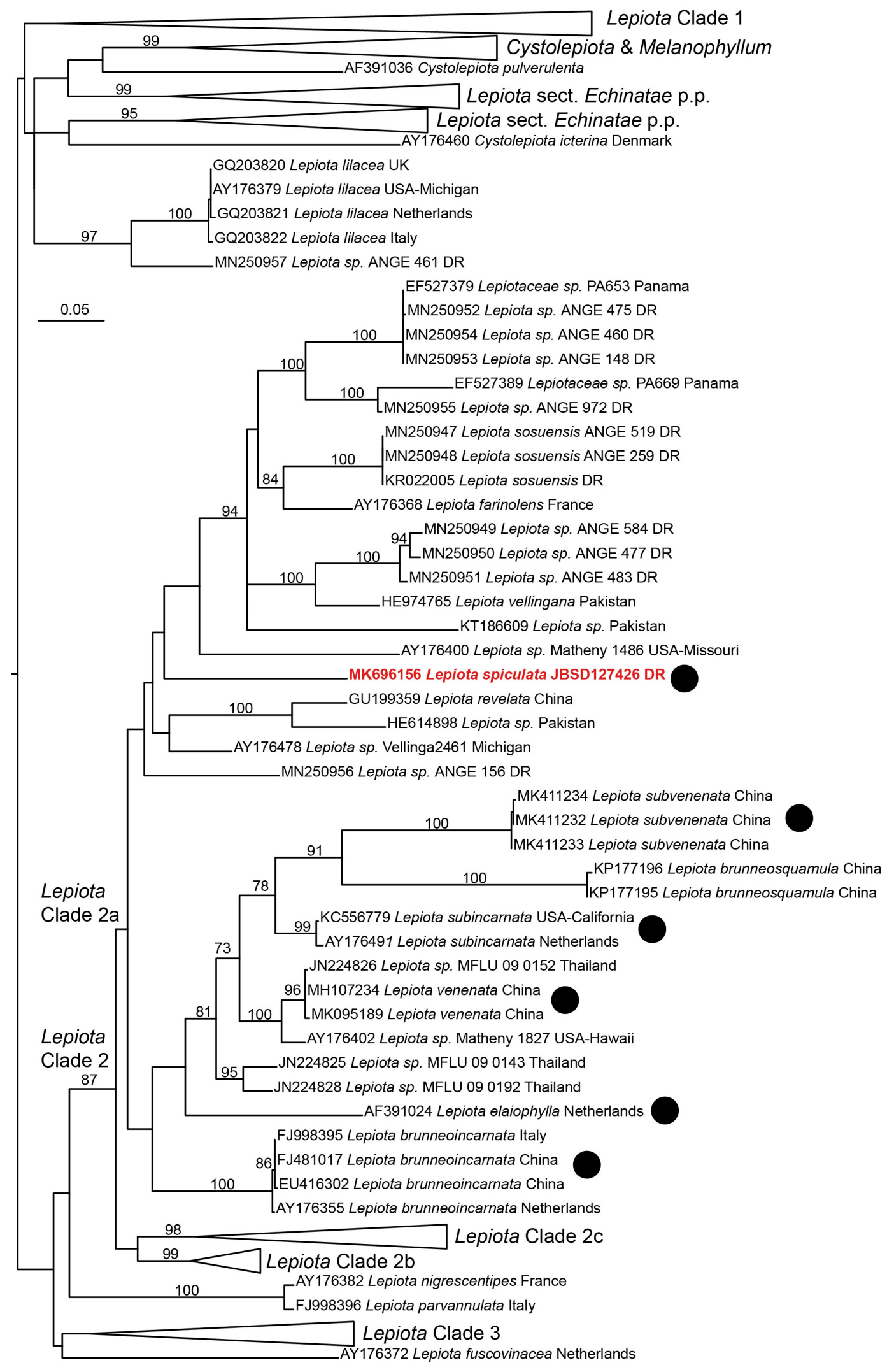

FIGURE 5 | Phylogenetic position of Lepiota spiculata based on a maximum likelihood analysis of an nrlTS dataset. Clade names follow Vellinga (2003). The black dots indicate taxa with confirmed presence of amatoxins. DR, Dominican Republic. 


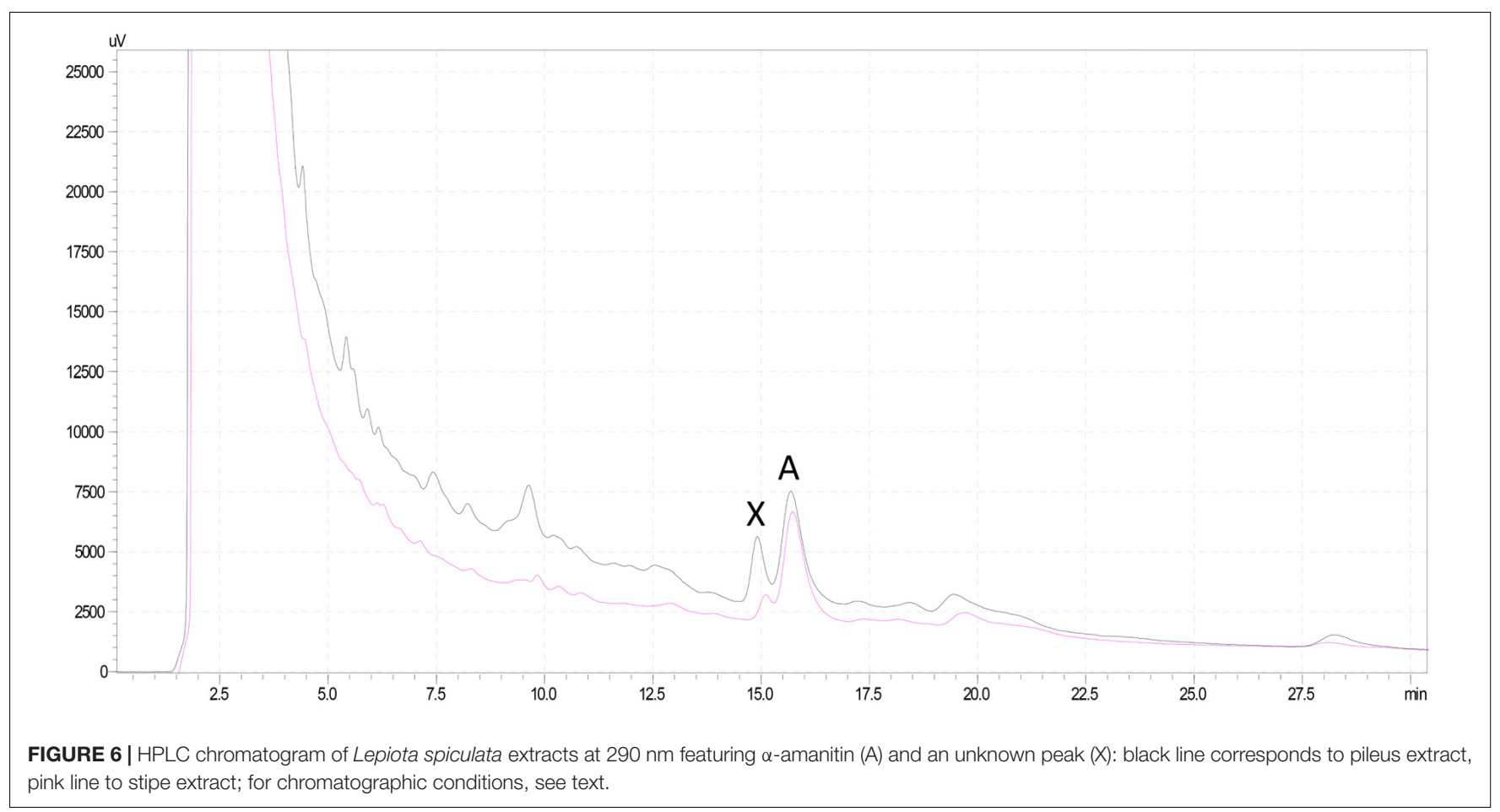

TABLE 2 | Toxin levels in Lepiota spiculata (in mg/g dry weight) as determined by HPLC analysis.

\begin{tabular}{lcccccc}
\hline & $\alpha$-Amanitin & $\beta$-Amanitin & $\gamma$-Amanitin & Phalloidin & Phallacidin & Unknown toxin* \\
\hline Pileus & $3.9 \pm 0.1$ & - & - & - & - & $1.2 \pm 0.1$ \\
Stipe & $2.7 \pm 0.1$ & - & - & - & - & $0.5 \pm 0.05$
\end{tabular}

*In the absence of an analytical standard, the concentration of the unknown toxin in L. spiculata is expressed as $\alpha$-amanitin equivalent.

Sgambelluri et al., 2014; Yilmaz et al., 2015; Walton, 2018). Although most Lepiota species of sect. Ovisporae (Vellinga, 2001; Liang et al., 2018) are smaller in size and are much less conspicuous and attractive in appearance (Razaq et al., 2013), they do account for fatal cases. In fact, although several Amanita species represent the most notorious source of amatoxins and are responsible for most fatal mushroom poisonings worldwide (Enjalbert et al., 2002; Tang et al., 2016; Wei et al., 2017; Diaz, 2018; Walton, 2018), fatal intoxications after ingestion of amatoxin-containing species of Lepiota also occur (Sgambelluri et al., 2014; Diaz, 2018). Lepiota poisonings have been reported in Europe, America, Asia, and North Africa (Tunisia) (Paydas et al., 1990; Watling, 1991; Ramirez et al., 1993; Khelil et al., 2010; Kervégant et al., 2013; Kose et al., 2015; Cai et al., 2018; Diaz, 2018; Sun et al., 2019), and the most frequently reported fatal cases are due to L. brunneoincarnata and L. subincarnata (Roux et al., 2008; Khelil et al., 2010; Mottram et al., 2010; Diaz, 2018; Sun et al., 2019). Lethal Lepiota species usually produce more than one type of amatoxin, for example, L. brunneoincarnata contains both $\alpha$ - and $\beta$-amanitin (Yilmaz et al., 2015), whereas $\alpha$ - and $\gamma$-amanitin are present in L. josserandii (Sgambelluri et al., 2014; Walton, 2018). By contrast, L. spiculata contains only $\alpha$-amanitin and L. venenata Zhu L. Yang \& Z.H. Chen, which has been recently responsible for fatal poisoning cases in China
(Cai et al., 2018), has been shown by analysis of genomic data to possess only the genes coding for $\alpha$-amanitin (Lüli et al., 2019). Species of the genus Galerina Earle also produce amatoxins at toxicologically relevant levels and are responsible for human and animal poisonings (Walton, 2018); in particular, wood-rotting basidiomes of G. marginata (Batsch) Kühner contain $\alpha-, \beta-$, and $\gamma$-amanitin at a combined level of $0.7-2.1 \mathrm{mg} / \mathrm{g}$ dry weight (Enjalbert et al., 2004; Walton, 2018), whereas $\alpha$ - and $\beta$-amanitin have been measured at a combined concentration up to $5.6 \mathrm{mg} / \mathrm{g}$ dry weight in Japanese specimens of G. helvoliceps (Berk. \& M.A. Curtis) Singer (Muraoka et al., 1999).

For the sake of comparison, concentrations of $\alpha$-amanitin in L. spiculata are higher than in Amanita phalloides (Fr.) Link, but the latter also contains other clinically relevant amatoxins such as $\beta$-amanitin that contribute to the overall amatoxin load (Walton, 2018). With regard to L. spiculata, therefore, the $\alpha$-amanitin levels that have been detected in the basidiomes are toxicologically relevant and render L. spiculata a potentially lethal species, if ingested. Hence, based on such data, even in the absence of poisoning reports L. spiculata must be listed accordingly among poisonous mushroom species.

It is desirable that the other few Lepiota species morphologically and/or molecularly assignable to section 

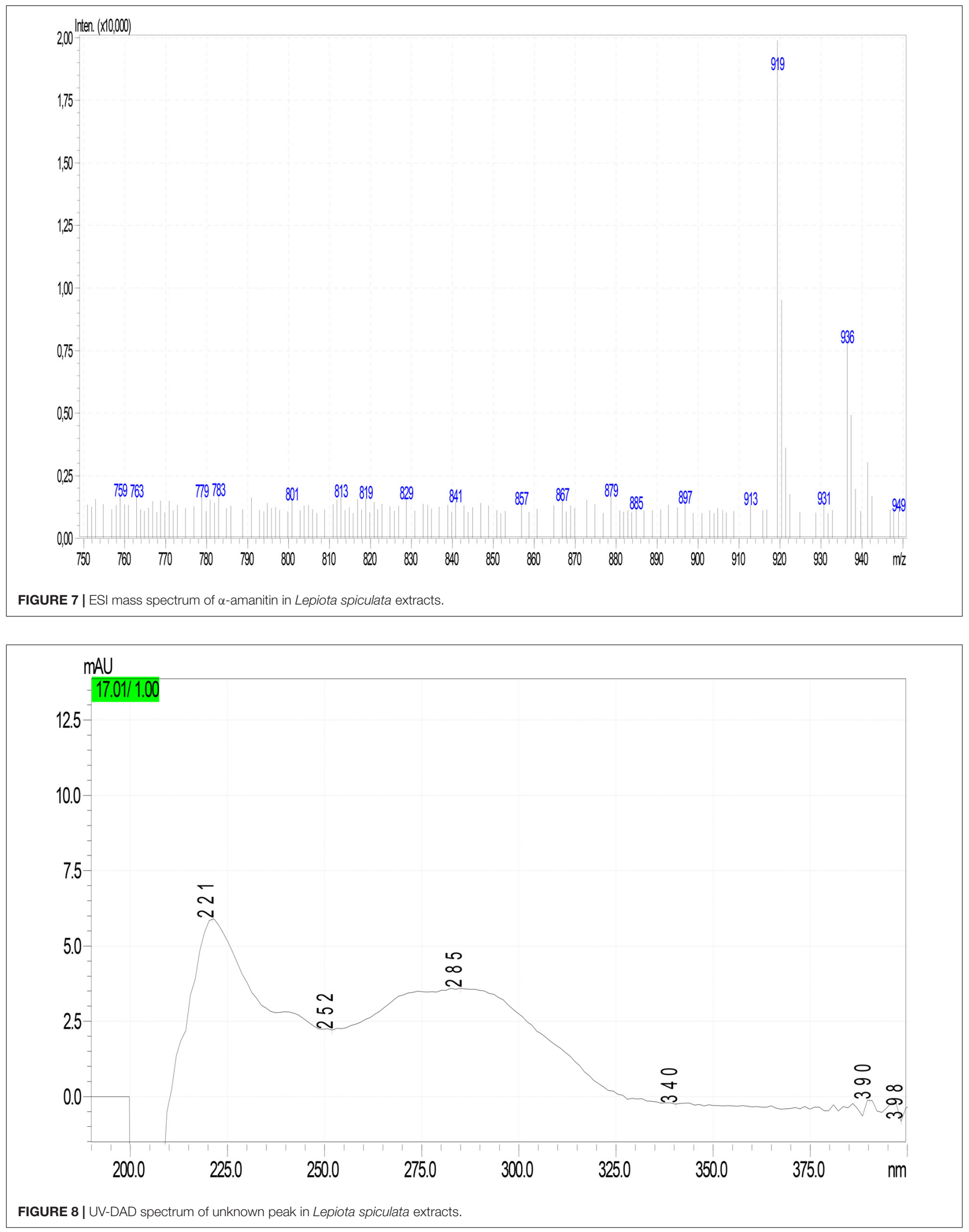
Ovisporae that have been described or reported from the Antilles (Caribbean area) (Pegler, 1983; Justo et al., 2015) will be investigated for the presence of amanitins.

\section{DATA AVAILABILITY STATEMENT}

The datasets generated for this study can be found in the GenBank, MK696156, MK696155, MK696577, and MK696576.

\section{AUTHOR CONTRIBUTIONS}

$\mathrm{CA}$ collected the species. $\mathrm{CA}$ and $\mathrm{AB}$ were responsible for the morphological analysis and description of the collection. AJ performed the molecular phylogenetic analyses. EK performed the chemical analysis. CA, AV, and PD planned, organized, and evaluated critically the experimental parts and wrote the

\section{REFERENCES}

Aanen, D. K., Ros, V. I. D., de Fine Licht, H. H., Mitchell, J., de Beer, Z. W., Slippers, B., et al. (2007). Patterns of interaction specificity of fungus-growing termites and Termitomyces symbionts in South Africa. BMC Evol. Biol. 7:115. doi: 10.1186/1471-2148-7-115

Baroni, T. (1998). Basidiomycetes of the Greater Antilles. Available online at: https://facultyweb.cortland.edu/NeoTropicalFungi/NSF/ga.asp (accessed on 20 January 2020).

Beutler, J. A., and Vergeer, P. P. (1980). Amatoxins in American mushrooms: evaluation of the Meixner test. Mycologia 72, 1142-1149. doi: 10.1080/ 00275514.1980.12021295

Cai, Q., Chen, Z. H., He, Z. M., Luo, H., and Yang, Z. L. (2018). Lepiota venenata, a new species related to toxic mushroom in China. J. Fungal Res. 16, 63-69.

Cubeta, M. A., Echandi, E., Abernethy, T., and Vilgalys, R. (1991). Characterization of anastomosis groups of binucleate Rhizoctonia species using restriction analysis of an amplified ribosomal RNA gene. Phytopathology 81, 1395-1400.

Diaz, J. H. (2018). Amatoxin-containing mushroom poisonings: species, toxidromes, treatments, and outcomes. Wilderness Environ. Med. 29, 111-118. doi: 10.1016/j.wem.2017.10.002

Enjalbert, F., Cassanas, G., Rapior, S., Renault, C., and Chaumont, J.-P. (2004). Amatoxins in wood-rotting Galerina marginata. Mycologia 96, 720-729. doi: 10.1080/15572536.2005.11832920

Enjalbert, F., Rapior, S., Nouguier-Soulé, J., Guillon, S., Amouroux, N., and Cabot, C. (2002). Treatment of amatoxin poisoning: 20-year retrospective analysis. J. Toxicol. Clin. Toxicol. 40, 715-757. doi: 10.1081/clt-12001 4646

Gardes, M., and Bruns, T. D. (1993). ITS primers with enhanced specificity for Basidiomycetes-application to the identification of mycorrhizae and rusts. Mol. Ecol. 2, 113-118. doi: 10.1111/j.1365-294x.1993.tb00005.x

Gicquel, T., Lepage, S., Fradin, M., Tribut, O., Duretz, B., and Morel, I. (2014). Amatoxins ( $\alpha$ - and $\beta$-Amanitin) and phallotoxin (Phalloidin) analyses in urines using high-resolution accurate mass LC-MS technology. J. Anal. Toxicol. 38, 335-340. doi: 10.1093/jat/bku035

Justo, A., Angelini, C., and Bizzi, A. (2015). Two new species and a new record of Lepiota (Basidiomycota, Agaricales) from the Dominican Republic. Mycol. Prog. 14:56.

Katoh, K., and Toh, H. (2008). Recent developments in the MAFFT multiple sequence alignment program. Brief Bioinform. 9, 286-298. doi: 10.1093/bib/ bbn013

Kervégant, M., De, H. L., Patat, A. M., Pons, C., Thomachot, L., and Minodier, P. (2013). Phalloides syndrome poisoning after ingestion of Lepiota mushrooms. Wilderness Environ. Med. 24, 170-172. doi: 10.1016/j.wem.2012.11.002 manuscript. All authors contributed to the article and approved the submitted version.

\section{ACKNOWLEDGMENTS}

We thank Else Vellinga (University of California, Berkeley, United States) for the determination of the species and for the valuable suggestions. We are thank to Luis A. Parra (Aranda de Duero, Burgos, Spain) for the revision of the nomenclature and for having contributed to the search for original material in European Herbaria, Pablo Alvarado (ALVALAB, Santander, Spain) for obtaining GenBank accession numbers, and Kurt Miller (UPRM, University of Puerto Rico, Mayagüez) for some suggestions in drafting the text in the English version. We are also very grateful to D. Jean Lodge (Department of Plant Pathology, University of Georgia, Athens, United States) for improving the English grammar.

Khelil, M. B., Zhioua, M., Bakir, O., Allouche, M., Gloulou, F., Banasr, A., et al. (2010). Intoxication mortelle par Lepiota brunneoincarnata: à propos de 4 cas. Ann. Biol. Clin. 68, 561-567. doi: 10.1684/abc.2010. 0467

Kose, M., Yilmaz, I., Akata, I., Kaya, E., and Guler, K. (2015). A case study: rare Lepiota brunneoincarnata poisoning. Wilderness Environ. Med. 26, 350-354. doi: 10.1016/j.wem.2014.12.025

Larsson, A. (2014). AliView: a fast and lightweight alignment viewer and editor for large datasets. Bioinformatics 30, 3276-3278. doi: 10.1093/bioinformatics/ btu531

Liang, J. F., Yu, F., Lu, J. K., Wang, S. K., and Song, J. (2018). Morphological and molecular evidence for two new species in Lepiota from China. Mycologia 110, 494-501. doi: 10.1080/00275514.2018.1464333

Liu, Y. J., Whelen, S., and Hall, B. D. (1999). Phylogenetic relationships among Ascomycetes: evidence from an RNA Polymerase II subunit. Mol. Biol. Evol. 16, 1799-1808. doi: 10.1093/oxfordjournals.molbev.a02 6092

Lüli, Y., Cai, Q., Chen, Z. H., Sun, H., Zhu, X.-T., Li, X., et al. (2019). Genome of lethal Lepiota venenata and insights into the evolution of toxin-biosynthetic genes. BMC Genomics 20:198. doi: 10.1186/s12864-019-5575-7

Mao, X. L. (2000). The Macrofungi in China. Zhengzhou: Henan Science \& Technology Press.

Matheny, P. B., Wang, Z., Binder, M., Curtis, J. M., Lim, Y. W., Nilsson, R. H., et al. (2007). Contributions of rpb2 and tef1 to the phylogeny of mushrooms and allies (Basidiomycota, Fungi). Mol. Biol. Evol. 43, 430-451. doi: 10.1016/j. ympev.2006.08.024

Meixner, A. (1979). Amatoxin-Nachweis in Pilzen. Z. Mykol. 45, 137-139.

Miller, M. A., Pfeiffer, W., and Schwartz, T. (2010). "Creating the CIPRES Science Gateway for inference of large phylogenetic trees," in Proceedings of the 10th Gateway Computing Environments Workshop (GCE), New Orleans, LA, 1-8.

Mottram, A. R., Lazio, M. P., and Bryant, S. M. (2010). Lepiota subincarnata J.E. Lange induced fulminant hepatic failure presenting with pancreatitis. J. Med. Toxicol. 6, 155-157. doi: 10.1007/s13181-010-0062-1

Muraoka, S., Fukamaki, N., Mizumoto, K., and Shinozawa, T. (1999). Detection and identification of amanitins in the wood-rotting fungi Galerina fasciculata and Galerina helvoliceps. Appl. Environ. Microbiol. 65, 4207-4210. doi: 10.1128/ aem.65.9.4207-4210.1999

Murray, M. G., and Thompson, W. F. (1980). Rapid isolation of high molecular weight plant DNA. Nucleic Acids Res. 8, 4321-4325.

Nobre, T., Rouland-Lefevre, C., and Aanen, D. K. (2011). "Comparative biology of fungus cultivation in termites and ants," in Biology of Termites: A Modern Synthesis, eds D. E. Bignell, Y. Roisin, and N. Lo (Cham: Springer International Publishing), 193-210. doi: 10.1007/978-90-481-3977-4_8 
Paydas, S., Kocak, R., Erturk, F., Erken, E., Zaksu, H. S., and Gurcay, A. (1990). Poisoning due to amatoxin-containing Lepiota species. Br. J. Clin. Pract. 44, 450-453.

Pegler, D. N. (1983). Agaric flora of the Lesser Antilles. London: Kew Bull. Add. Ser. Ramirez, P., Parrilla, P., Sanchez Bueno, F., Robles, R., Pons, J. A., Bixquert, V., et al. (1993). Fulminant hepatic failure after Lepiota mushroom poisoning. J. Hepatol. 19, 51-54. doi: 10.1016/s0168-8278(05)80175-8

Razaq, A., Vellinga, E. C., Ilyas, S., and Khalid, A. N. (2013). Lepiota brunneoincarnata and L. subincarnata: distribution and phylogeny. Mycotaxon 126, 133-141. doi: 10.5248/126.133

Rehner, S. A., and Buckley, E. (2005). A Beauveria phylogeny inferred from nuclear ITS and EF1-a sequences: evidence for cryptic diversification and links to Cordyceps teleomorphs. Mycologia 97, 84-98. doi: 10.3852/mycologia.97.1.84

Roux, X., Labadie, P., Morand, C., Fontaine, B., and Coutant, G. (2008). Mushroom poisoning by Lepiota brunneoincarnata: about two cases. Ann. Fr. Anesth. Reanim. 27, 450-452.

Séguy, E. (1936). Code Universal des Couleurs, VI Edn. Paris: Paul Lechevalier.

Sgambelluri, R. M., Epis, S., Sassera, D., Luo, H., Angelos, E. R., and Walton, J. D. (2014). Profiling of amatoxins and phallotoxins in the genus Lepiota by liquid chromatography combined with UV absorbance and mass spectrometry. Toxins 6, 2336-2347. doi: 10.3390/toxins6082336

Singer, R., and Garcia, J. (1989). Two interesting fungi of the Western Hemisphere. Mycologist 3, 70-71. doi: 10.1016/s0269-915x(89)80079-5

Stamatakis, A. (2014). RAxML version 8: a tool for phylogenetic analysis and post-analysis of large phylogenies. Bioinformatics 30, 1312-1313. doi: 10.1093/ bioinformatics/btu033

Sun, J., Zhang, H.-S., Li, H.-J., Zhang, Y.-Z., He, Q., Lu, J.-J., et al. (2019). A case study of Lepiota brunneoincarnata poisoning with endoscopic nasobiliary drainage in Shandong, China. Toxicon 161, 12-16. doi: 10.1016/j.toxicon.2019. 02.017

Sysouphanthong, P., Hyde, K. D., Chukeatirote, E., and Vellinga, E. C. (2011). A review of genus Lepiota and its distribution in Asia. Curr. Res. Environ. Appl. Mycol. 1, 161-176.

Tang, S., Zhou, Q., He, Z., Luo, T., Zhang, P., Cai, Q., et al. (2016). Cyclopeptide toxins of lethal amanitas: compositions, distribution and phylogenetic implication. Toxicon 120, 78-88. doi: 10.1016/j.toxicon.2016.07.018

Thiers, B. (2020). Index Herbariorum: A Global Directory of Public Herbaria and Associated Staff. New York Botanical Garden's Virtual Herbarium. (Continuously Updated). Available from: http://sweetgum.nybg.org/ih/ (accessed on 28 January 2020).

Vellinga, E. C. (1988). “Glossary,” in Flora Agaricina Neerlandica, Vol. 1, eds C. Bas, T. W. Kuyper, M. E. Noordeloos, and E. C. Vellinga (Rotterdam: A.A. Balkema), 54-64.

Vellinga, E. C. (2001). "Lepiota (Pers.: Fr.) S.F.Gray," in Flora Agaricina Neerlandica, Vol. 5, eds M. E. Noordeloos, T. W. Kuyper, and E. C. Vellinga (Lisse: A.A. Balkema Publishers), 109-150.

Vellinga, E. C. (2003). Phylogeny of Lepiota (Agaricaceae) - evidence from nrITS and nrLSU sequences. Mycol. Prog. 2, 305-322. doi: 10.1007/s11557-0060068-x

Vellinga, E. C. (2004a). Ecology and distribution of lepiotaceous Fungi (Agaricaceae) - a review. Nova Hedwigia 78, 273-299. doi: 10.1127/0029-5035/ 2004/0078-0273
Vellinga, E. C. (2004b). Genera in the family Agaricaceae: evidence from nrITS and nrLSU sequences. Mycol. Res. 108, 354-377. doi: 10.1017/s095375620400 9700

Vilgalys, R., and Hester, M. (1990). Rapid genetic identification and mapping of enzymatically ampliWed ribosomal DNA from several Cryptococcus species. J. Bacteriol. 172, 4238-4246. doi: 10.1128/jb.172.8.4238-4246.1990

Visser, A. A., Kooij, P. W., Debets, A. J. M., Kuyper, T. W., and Aanen, D. K. (2011). Pseudoxylaria as stowaway of the fungus-growing termite nest: interaction asymmetry between Pseudoxylaria, Termitomyces and free-living relatives. Fungal Ecol. 4, 322-332. doi: 10.1016/j.funeco.2011.05.003

Walton, J. (2018). The Cyclic Peptide Toxins of Amanita and other Poisonous Mushrooms. Cham: Springer International Publishing.

Watling, M. I. R. (1991). Poisonings by Lepiota helveola Bres. in southern Turkey. Edinb. J. Bot. 48, 91-100. doi: 10.1017/s0960428600003668

Wei, J., Wu, J., Chen, J., Wu, B., He, Z., Zhang, P., et al. (2017). Determination of cyclopeptide toxins in Amanita subpallidorosea and Amanita virosa by high-performance liquid chromatography coupled with high-resolution mass spectrometry. Toxicon 133, 26-32. doi: 10.1016/j.toxicon.2017.04.012

White, T. J., Bruns, T. D., Lee, S., and Taylor, J. W. (1990). “Amplification and direct sequencing of fungal ribosomal RNA genes for phylogenetics," in PCR Protocols: A Guide to Methods and Applications, eds M. A. Innis, D. H. Gelfand, J. Sninsky, and T. J. White (New York: Academic Press), 315-322. doi: 10.1016/b978-0-12372180-8.50042-1

Ye, L., Karunarathna, S. C., Li, H., Xu, J., Hyde, K. D., and Mortimer, P. E. (2019). A survey of Termitomyces (Lyophyllaceae, Agaricales), including a new species, from a subtropical forest in Xishuangbanna, China. Mycobiology 47, 391-400. doi: 10.1080/12298093.2019.1682449

Yilmaz, I., Akata, I., Bakirci, S., and Kaya, E. (2018). Lepiota cristata does not contain amatoxins or phallotoxins. Toxin Rev. 37, 149-153. doi: 10.1080/ 15569543.2017.1337034

Yilmaz, I., Bakirci, S., Akata, I., Bayram, R., and Kaya, E. (2015). Toxin content and toxicological significance in different tissues and development stages of Lepiota brunneoincarnata mushroom. Toxin Rev. 34, 109-114. doi: 10.3109/15569543. 2015.1072563

Yilmaz, I., Sinirlioglu, Z. A., Karahan, S., Bayram, R., Yaykasli, K. O., Colakoglu, S., et al. (2013). Amanitin and phallotoxin concentration in Amanita phalloides var. alba mushroom. Toxicon 76, 225-233. doi: 10.1016/j.toxicon.2013.10.008

Zhang, Y.-Z., Zhang, K.-P., Zhang, H.-S., Sun, J., Yin, Y., Li, H.-J., et al. (2019). Lepiota subvenenata (Agaricaceae, Basidiomycota), a new poisonous species from southwestern China. Phytotaxa 400, 265-272.

Conflict of Interest: The authors declare that the research was conducted in the absence of any commercial or financial relationships that could be construed as a potential conflict of interest.

Copyright (C) 2020 Angelini, Vizzini, Justo, Bizzi, Davoli and Kaya. This is an openaccess article distributed under the terms of the Creative Commons Attribution License (CC BY). The use, distribution or reproduction in other forums is permitted, provided the original author(s) and the copyright owner(s) are credited and that the original publication in this journal is cited, in accordance with accepted academic practice. No use, distribution or reproduction is permitted which does not comply with these terms. 